\title{
SAHUARUS
}

Revista Electrónica de Matemáticas,

Departamento de Matemáticas, Universidad de Sonora,

Vol. 1, No. 1, Enero 2016, pp. 51-57.

\section{Resolución de problemas en el bachillerato: las estrategias de los profesores}

\section{Arcelia Cecilia Moreno Verdugo José Luis Soto Munguía}

\author{
Departamento de Matemáticas, Universidad de Sonora \\ e-mail: arcelia103@outlook.com
}

\begin{abstract}
Resumen
En este artículo se presenta un estudio acerca de las estrategias que utilizan los profesores al resolver problemas; la investigación se desarrolla con profesores del Colegio de Bachilleres del Estado de Sonora (COBACH-SON). Este trabajo se hace de acuerdo con las categorías propuestas por Schoenfeld, para entender el conocimiento y las actitudes de los profesores de bachillerato al resolver problemas geométricos. Se consideran principalmente las categorías de heurísticas y control.
\end{abstract}

\section{Introducción}

La resolución de problemas es la esencia de las matemáticas, y lo ha sido a lo largo de su desarrollo, pues desde tiempos remotos los problemas han sido el motor para la generación y el refinamiento de los conceptos matemáticos. Sin embargo la importancia conferida a la resolución de problemas matemáticos en el salón de clases no se corresponde ni con el papel que los problemas han jugado en el desarrollo histórico de las matemáticas, ni con el énfasis declarado por las instituciones al respecto. En el presente trabajo se reporta una investigación en curso, que tiene como objetivo analizar las estrategias usadas por el profesor como resolutor de problemas. El trabajo está centrado en los problemas geométricos, ya que en ellos las estrategias son más claramente observables durante el proceso de resolución.

\section{Marco conceptual}

El trabajo se basa en las contribuciones teóricas de Schoenfeld $[\mathbf{2 , 3}, \mathbf{4}, \mathbf{5}, \mathbf{6}]$, en donde refina la metodología para la resolución de problemas propuesta por Polya [1], principalmente las heurísticas, y clasifica en cuatro categorías el conocimiento y las actitudes necesarias en la resolución de problemas. Éstas son: los recursos, las heurísticas, el control y los sistemas de creencias. Por una parte, cuando se habla de recursos se refiere a los conocimientos matemáticos que poseen las personas y que pueden ser empleados al resolver un problema. Por otro lado, las creencias están constituidas por lo que se piensa acerca de las matemáticas, de uno mismo, etc, e influyen en las decisiones que se toman durante el proceso de resolución. Es preciso mencionar que este trabajo se centra principalmente en las heurísticas y el control, por lo que estas categorías son explicadas con más detalle en las secciones siguientes. 
MORENO, SOTO

\subsection{Heurísticas}

Cuando se tiene un problema por resolver usualmente no se cuenta con una estrategia determinada para encontrar su solución, es aquí donde se percibe el papel que juegan las heurísticas. Éstas son técnicas o sugerencias que sirven para comprender mejor un problema, y con frecuencia permiten resolverlo. Con el fin de que las heurísticas propuestas en [2] fueran más fáciles de comprender y utilizar, Schoenfeld [2 , p. 109] desglosa las heurísticas utilizadas frecuentemente para resolver problemas. Éstas son: el análisis, la exploración y la verificación de la solución. Primeramente, cuando se quiere resolver un problema se comienza por leerlo cuidadosamente y se hace un análisis de éste para entender los requerimientos, datos o contexto del problema; así como para determinar qué heurística se va a utilizar para resolver el problema. Algunas acciones (también consideradas como heurísticas) que se realizan en el análisis de un problema son: dibujar figuras, considerar casos especiales y simplificar el problema.

Luego está la fase de exploración, que es donde las estrategias de resolución de problemas entran en juego; algunas de estas estrategias son: considera problemas modificados, considerar problemas equivalentes, etc. Una vez terminada la fase de exploración, quien resuelve el problema puede volver a hacer un análisis del problema para obtener una perspectiva diferente a la que se analizó el problema, en un primer momento. Otra estrategia es la de verificación de la solución, que como su nombre lo dice se trata de revisar el proceso de resolución para identificar "errores de dedo", para encontrar otra manera de resolver el problema, o bien para considerar la posibilidad de usar la solución al resolver otros problemas.

\subsection{Control}

Polya [2] consideraba las heurísticas como el único factor que influía en la resolución de problemas, sin embargo, Schoenfeld en [2] propuso por primera vez otra categoría que podría definir el éxito o el fracaso al intentar resolver un problema: el control. Definió esta categoría como las decisiones globales respecto a la selección e implementación de recursos y estrategias [2, p. 15], y cabe mencionar que la toma oportuna de decisiones es considerada como la esencia de la inteligencia; ya no importa solamente lo que sabe quien resuelve el problema, sino cómo y cuándo usa lo que sabe. Algunas habilidades referidas al control pueden ser la planificación, la supervisión, la toma de decisiones y los actos conscientes metacognitivos. La planificación se refiere a elaborar un plan de todo el proceso de resolución; la supervisión se trata de revisar con detalle cada procedimiento o etapa del proceso.

La toma de decisiones tiene que ver, por ejemplo, con determinar en qué momento se termina del proceso de resolución, cuándo abandonarlo, cambiar de estrategia, etc. Por último están los actos conscientes metacognitivos, que se refiere a que un individuo se corrija a sí mismo y reflexione acerca de sus reflexiones personales, y que esté consciente de lo que está haciendo. Además, Schoenfeld [2, p.116] muestra cuatro tipos de comportamiento en la resolución de problemas, los cuales son: 
- Tipo A. Malas decisiones garantizan fracaso: Búsqueda inútil, se desperdician los recursos y se ignoran las direcciones potencialmente útiles.

- Tipo B. Comportamiento directivo es neutral: Búsquedas inútiles se reducen antes de causar desastres, pero los recursos no están siendo explotados como podrían serlo.

- Tipo C. Las decisiones de control son una fuerza positiva en la solución: Los recursos son cuidadosamente elegidos y explotados o abandonados adecuadamente como resultado de un cuidadoso monitoreo.

- Tipo D. No hay necesidad de un comportamiento de control: Los hechos y procedimientos apropiados para la resolución de problemas son accedidos en la memoria a largo plazo (LTM, por sus siglas en inglés).

\section{Método}

Para lograr el objetivo de este trabajo, se considera una etapa que consiste identificar las estrategias que emplean los maestros para resolver problemas de geometría. El propósito de esta etapa consistió en indagar acerca de: ¿Qué hace antes de comenzar a resolver un problema?, ¿elabora un plan de solución?, ¿qué estrategias utiliza para resolver problemas?, ¿qué flexibilidad tiene el profesor para abandonar una estrategia fallida, así como para emplear otra estrategia en el intento de resolver el problema?, ¿Cómo son las estrategias que utiliza respecto a las que enseña en el salón de clases?, entre otras. Con la intención de recabar la información correspondiente dicha fase, se seleccionaron dos profesores de COBACH y se eligió el siguiente instrumento:

1. Entrevista a los profesores. La entrevista tiene un diseño semiestructurado, y durante su desarrollo se plantearon al profesor los problemas del cuestionario, procurando que sus estrategias de solución quedaran explícitas. Por esta razón, se solicitó al entrevistado que durante el proceso de resolución "piense en voz alta". Para recabar la mayor cantidad de datos sobre las estrategias de solución, tanto los registrados en papel como los externados por el profesor al "pensar en voz alta", las entrevistas fueron videograbadas. El problema que se les planteó a los profesores es el siguiente:

\section{Parte experimental}

Se realizaron varias pruebas piloto de la entrevista, con estudiantes de la Licenciatura en Matemáticas de la Universidad de Sonora; éstas tuvieron un carácter exploratorio y sirvieron para adquirir familiaridad con este tipo de entrevista. Las pruebas piloto de las entrevistas han tenido una duración aproximada de una hora, y el entrevistador ha intervenido cada vez que alguna acción del estudiante no ha quedado clara, principalmente aquellas relacionadas con las heurísticas y el control. Los resultados preliminares arrojados por las entrevistas evidencian que es posible identificar durante el proceso de resolución de un problema, las estrategias usadas por el resolutor, así como las habilidades referidas al control. Ahora, ya 


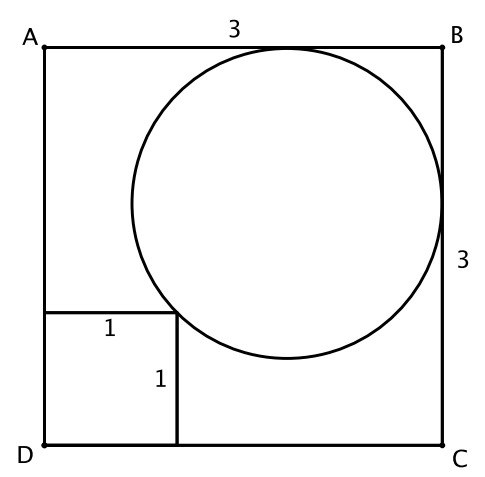

Figura 1:

se entrevistó a los profesores y se hace un análisis del proceso de resolución; a continuación se presenta el análisis de un extracto de la entrevista de la profesora $\mathbf{M}$.

\subsection{Análisis del extracto}

Al comenzar el proceso de resolución, M leyó el problema, exploró e identificó en la Figura 1 los datos que le proporcionaba el enunciado, ya fueran explícitos (por ejemplo las medidas de los lados de los cuadrados) o implícitos (la diagonal del cuadrado de lado uno o el segmento de medida dos; ver Figura 2). M se encontraba en la etapa de análisis del problema y pareciera que las exploraciones que hizo tienen que ver con la idea de reunir elementos o trazos auxiliares que le ayuden a ubicar el radio, pero no alcanzó a situar el radio o a conjeturar como está relacionado con los datos. Después y durante aproximadamente un minuto continúo haciendo exploraciones en el dibujo; trazó una cuerda, una línea que pasaba por el centro y dibujó el centro (ver Figura 3). Da la impresión de que diseñó un plan, o bien que estableció un subobjetivo (o subproblema): encontrar un radio a partir de las relaciones entre el centro y los trazos auxiliares.

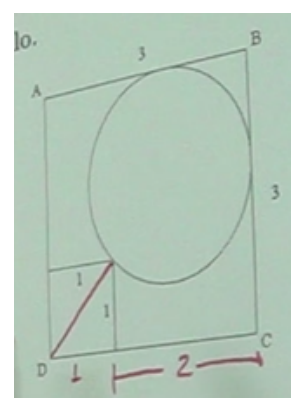

Figura 2:

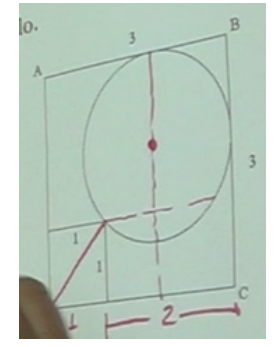

Figura 3:

Hasta este momento, $\mathbf{M}$ estaba orientando la resolución del problema, ya que se fijó un subobjetivo y las intervenciones de los entrevistadores eran mínimas y no influenciaron 
las acciones de M. No obstante, la iniciativa de encontrar un radio y las dificultades para ubicarlo, le exigía seguir haciendo exploraciones en éste que le permitieran visualizar por lo menos un radio y establecer un punto de partida para el cálculo de la medida del radio. En la búsqueda del radio, ella procede de la siguiente manera:

M: Ésta pasa por el centro [la línea paralela a $B C$ ] ... entonces éste vale uno y éste también vale uno [anota las medidas de las mitades de la cuerda, y traza el radio de la circunferencia como prolongación de la diagonal del cuadrado de lado uno (ver Figura 4)] ... aaah ...mmh ... éste también vale uno [señala el lado del cuadrado de lado uno] ... muy bien ...mmh ... [hace un silencio de 5 segundos].

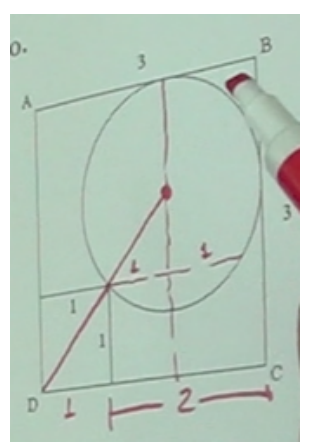

Figura 4:

$\mathbf{E}_{1}$ : Esos trazos los está haciendo con la idea de buscar el centro?

M: Mmh, si pues, para buscar cómo está relacionado pues las medidas y decir dónde puedo yo encontrar pues la relación ... la relación de esta medida [el lado del cuadrado de lado uno] si conozco la completa [el lado del cuadrado $A B C D$ ], y ver esteee, cómo se relaciona con el diámetro, con el centro no?, de la circunferencia, pues, nos está pidiendo el radio no? ... el radio ...mmh . . éste no vale uno, y éste tampoco [tacha los valores de los segmentos de la cuerda (ver Figura 5)] ... [hace un silencio de 7 segundos].

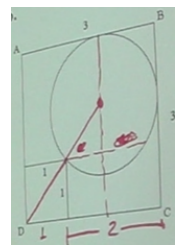

Figura 5: 
Cuando $\mathbf{M}$ dice "Ésta pasa por el centro ... entonces éste vale uno y éste también vale uno", estaba conjeturando acerca de que el segmento paralelo a $B C$ y que pasa por el centro de la circunferencia divide en dos partes iguales a la cuerda que trazó. En la búsqueda de recursos, $\mathbf{M}$ considera una falsa conjetura, y a pesar de que se observa que la cuerda no mide dos, no cae en cuenta del error; la suposición que hizo requería de una verificación acerca de la consistencia de los datos, la cual no percibió en este momento del proceso. Las acciones hechas por $\mathbf{M}$ se pueden clasificar dentro del comportamiento de Tipo $\mathbf{A}$, definido por [1] como "Malas decisiones garantizan fallos". Se considera ese tipo de comportamiento, porque ella considera, en un primer momento, que la paralela a $B C$ que pasa por el centro divide en dos partes iguales al segmento en $C D$ de medida dos, lo cual es falso y la lleva a suponer que las medidas de las mitades de la cuerda miden uno; teniendo como consecuencia dos supuestos falsos a partir de la búsqueda de recursos que hace. Además de no tener una justificación de por qué dichos valores valen uno (más allá de la visualización), por lo que es posible que ella tomara una dirección hacia una solución incorrecta del problema.

Quizá con esta conjetura, M pretendía encontrar una relación entre la cuerda (o bien, la mitad de la cuerda) y el radio, y tal vez la conclusión de que tales segmentos medían uno le resolvía el problema; aunque segundos después, como puede verse en su segunda intervención, se observa la relación que está buscando. Cuando dice: "puedo encontrar ... la relación de esta medida si conozco la completa", posiblemente se refiere a encontrar la medida del diámetro a partir de las medidas del cuadrado de lado uno y del cuadrado $A B C D$, aunque pareciera no saber cómo encontrar dicha relación. En este momento da la impresión de que ya no está considerando tomar en cuenta las mitades de la cuerda, pero cabe la posibilidad de que esté buscando una relación, que le sea útil, entre el radio, la cuerda y las medidas antes mencionadas. Otra relación que pudo considerar, tiene que ver con lo que resulta al unir los trazos de la cuerda, el radio y la línea paralela a $B C$; se forma un triángulo rectángulo (véase la Figura ??), del cual $\mathbf{M}$ conoce la supuesta medida de los catetos (que es uno) y de la hipotenusa (el radio). Sin embargo, no alcanza a usar o visualizar dicha relación, porque se da cuenta de que los valores no son correctos.

\section{Conclusiones}

En la resolución de problemas el control es un mecanismo que define el rumbo de este proceso, sin embargo está ampliamente relacionado con las heurísticas. En el extracto que se presenta y analiza, el cual forma parte del proceso de resolución llevado acabo por la profesora $\mathbf{M}$, se identifican distintas heurísticas y habilidades referidas el control. Respecto a las heurísticas, en el análisis se percibe que ella recurre con más frecuencia a la exploración, en particular con introducir trazos auxiliares; lo cual es habitual en la resolución de problemas geométricos.

De acuerdo con la categoría de control, la presencia y la ausencia de las habilidades referidas a éste se perciben a lo largo del extracto. Por ejemplo, ella accede a los principios cuando conjetura que "Ésta pasa por el centro ....entonces éste vale uno y éste también 
vale uno", sin embargo ésta es falsa y no regresa a reconstruir la relación que establece, lo cual tiene que ver con de revisar la estrategia para remediar las dificultades, el cual es un mecanismo de control. Asimismo, la toma de decisiones entra en acción cuando las cosas no parecen ir bien, o cuando $\mathbf{M}$ no tiene la seguridad de implementar o abandonar una estrategia; no obstante, las orientaciones que ella le da al proceso, no necesariamente la llevan al éxito en la resolución del problema.

\section{Referencias}

[1] Polya, G., Cómo plantear y resolver problemas, 215, Trillas, México (1965)

[2] Schoenfeld, A., Mathematical Problem Solving, 409, Academic Press, Orlando (1985)

[3] Schoenfeld, A., Learning to think mathematically: Problem solving, metacognition and sense-making in mathematics, In D. Grouws (Ed.), Handbook for Research on Mathematics Teaching and Learning, pp. 334-370 (1992)

[4] Schoenfeld, A., Toward a theory of teaching-in-context, Issues in Education, Volume 4, Number 1, pp. 1-94 (1998)

[5] Schoenfeld, A., Problem solving, teaching, and more: Toward a theory of goal-directed behavior. CIEAEM, Volume 59, pp. 48-52 (2007)

[6] Schoenfeld, A., How We Think: A Theory of Goal-Oriented Decision Making and its Educational Applications, 491, Routledge, New York (2011) 